\title{
DYADIC FEFFERMAN-STEIN INEQUALITIES AND THE EQUIVALENCE OF HAAR BASES ON WEIGHTED LEBESGUE SPACES
}

\author{
HUGO AIMAR, ANA BERNARDIS, AND LUIS NOWAK
}

\begin{abstract}
In this note we give sufficient conditions on two dyadic systems to obtain the equivalence of corresponding Haar systems on dyadic weighted Lebesgue spaces on spaces of homogeneous type. In order to obtain these result we prove a Fefferman-Stein weighted inequality for vector valued dyadic HardyLittlewood maximal operators with dyadic weights in this general setting.
\end{abstract}

\section{INTRODUCTION}

One of the basic aims of the so called wavelet theory is the design of bases for the space $L^{2}$ with as much good properties as possible. In particular the metric control of the supports of the wavelets seems an important property to preserve.

If the space $L^{2}$ which we explore is defined on an abstract metric space $(X, d)$, for which no smoothness better than Lipschitz continuity makes sense, the first basic prototype of localized wavelet is the Haar wavelet.

It is easy to built a Haar type basis when an adequate sequence of nested partitions of the whole space is given. If the supports of such wavelets are expected to be concentrated in some regions of the space in the metric sense, we need to have partition with metric control.

Nested measurable partitions of measurable spaces are usually considered in harmonic analysis and probability. The conditional expectations of a random variable with respect to the sequence of $\sigma$-algebras generated by each partition gives rise to the concept of martingale. On the other hand the basic algorithm of the CalderónZygmund decomposition of the Euclidean space for a given integrable function relies on such partition process. The dyadic cubes involved in the Calderón-Zygmund technique have the advantage of the metric control.

The first non-trivial construction of such partitions with a complete metric control is provided by M. Christ in [9] in the setting of spaces of homogeneous type. In [4] we define dyadic families on a space of homogeneous type with metric control. These families contain at once the classical dyadic cubes in an Euclidean environment and those Christ's families.

There we give sufficient conditions on two different dyadic systems on the same space of homogeneous type, in such a way that Haar bases built on them become equivalent on Lebesgue spaces. The equivalence of bases here is taken in the sense

2000 Mathematics Subject Classification. 42C15, 42B20, 28C15 .

Key words and phrases. Fefferman-Stein inequalities, Haar basis, equivalence of bases, spaces of homogeneous type. 
of equivalence of bases in Banach spaces given by [12] and [18]. Actually in [4] we show that under a certain notion of equivalence of two dyadic families we obtain the corresponding equivalence of Haar systems on $L^{p}(w)$ for weights $w$ in the Muckenhoupt $A_{p}(1<p<\infty)$ class defined on the abstract space of homogeneous type. The central tool there is the use of Fefferman-Stein weighted inequality for vector valued Hardy-Littlewood maximal operators ([11]), aside from the characterization of weighted Lebesgue spaces through the Haar coefficient ([3]).

Since we are mostly dealing with dyadic analysis, the result obtained in [4] is somehow restricted. In fact, the natural classes of weights for the equivalence of Haar systems in $L^{p}(w)$ are those of the dyadic Muckenhoupt weights, $A_{p}^{\mathcal{D}}$. As in the Euclidean case these classes are typically larger than the standard $A_{p}$ classes.

In this paper we obtain sufficient conditions on two dyadic families to get the equivalence of associated Haar systems in $L^{p}(w)$ with $w \in A_{p}^{\mathcal{D}}$ and $1<p<\infty$.

The condition of equivalence of the dyadic families is strictly stronger that the one in [4]. The basic tool is now a Fefferman-Stein weighted inequality for dyadic vector valued Hardy-Littlewood maximal operators with dyadic weights in spaces of homogeneous type.

The paper is organized as follows. In Section 2 we introduce the basic concepts of the general theory of space of homogeneous type and recall the definition of dyadic families. We also give in this section the concept of equivalence of dyadic systems that shall be used in the main result. Here we show that different Christ's families with the same sequence of nets are equivalent in this sense. In Section 3 we prove that Haar systems associated to two equivalent dyadic families are equivalent, in the sense of equivalence of Schauder bases in Banach spaces, in dyadic weighted Lebesgue spaces. As particular case, we obtain the equivalence of Haar bases built on equivalent Christ's dyadic families. Section 4 is dedicated to prove a dyadic weighted version of the Fefferman-Stein inequalities for the dyadic HardyLittlewood vector valued maximal operator in the context of space of homogeneous type.

\section{EquivalenCE OF DYADIC SYSTEMS}

Assume that $X$ is a set, a nonnegative symmetric function $d$ on $X \times X$ is called a quasi-distance if there exists a constant $K$ such that

$$
d(x, y) \leq K[d(x, z)+d(z, y)]
$$

for every $x, y, z \in X$, and $d(x, y)=0$ if and only if $x=y$.

We shall say that $(X, d, \mu)$ is a space of homogeneous type if $d$ is a quasi-distance on $X, \mu$ is a positive Borel measure defined on a $\sigma$-algebra of subsets of $X$ which contains the balls, and there exists a constant $A$ such that

$$
0<\mu(B(x, 2 r)) \leq A \mu(B(x, r))<\infty
$$

holds for every $x \in X$ and every $r>0$.

The sets $\{(x, y) \in X \times X: d(x, y)<1 / n\}$ define a basis of a metrizable uniform structure on $X$. The balls $B(x, r)=\{y: d(x, y)<r\}$ form a basis of neighborhoods of $x$ for the topology induced by the uniform structure. A basic caveat comes from the fact that the $d$-balls are generally not open sets. Moreover, it is not difficult to 
give examples of quasi-distances for which some balls are not even Borel measurable subsets of $X$. Nevertheless in [13], R. Macias and C. Segovia prove that if $d$ is a quasi-distance on $X$, then there exist a distance $\rho$ and a number $\alpha \geq 1$ such that $d$ is equivalent to $\rho^{\alpha}$. Hence except for some simple changes of scales we can keep working with $\rho$ instead of $\rho^{\alpha}$. So that we shall assume along this paper that $d$ is actually a distance on $X$, in other words that $K=1$ in (2.1).

We shall say that a subset $E$ of $X$ is $\epsilon$-disperse for $\epsilon>0$ if $d(x, y) \geq \epsilon$ for every $x, y$ in $E$ with $x \neq y$.

Given a set $E$ in $X$ we shall write $\operatorname{diam}(E)$ to denote its diameter. In other words, $\operatorname{diam}(E)=\sup \{d(x, y): x \in E, y \in E\}$. On the other hand given an open and bounded set $E$ in $X$ we define its eccentricity by $\varepsilon(E)=\frac{r_{i}(E)}{r_{o}(E)}$, where $r_{i}(E)=$ $\sup \{r: B(x, r) \subseteq E$ for some $x \in E\}$ and $r_{o}(E)=\inf \{r: E \subseteq B(x, r)$ for some $x \in$ $X\}$.

In order to be able to apply Lebesgue Differentiation Theorem we shall also assume that continuous functions are dense in $L^{1}(X, \mu)$. Under the assumption of completeness for $(X, d)$ and regularity of the measure $\mu$ this density can be achieved (see [14] and [15]).

As we mentioned in the introduction, a construction of dyadic type families of subsets in metric or quasi-metric measure spaces $(X, d, \mu)$, with some inner and outer metric control of the sizes of the dyadic sets, is given in [9]. This result shows that the classes introduced in the next definition are non trivial in every space of homogeneous type.

Definition 2.1. The class $\mathfrak{D}(\delta)$ of all dyadic families. Let $(X, d, \mu)$ be a metric space of homogeneous type. We say that $\mathcal{D}=\bigcup_{j \in \mathbb{Z}} \mathcal{D}^{j}$ is a dyadic family on $X$ with parameter $\delta \in(0,1)$, briefly that $\mathcal{D}$ belong $\mathfrak{D}(\delta)$, if each $\mathcal{D}^{j}$ is a family of open subsets $Q$ of $X$, such that

(d.1) For every $j \in \mathbb{Z}$ the cubes in $\mathcal{D}^{j}$ are pairwise disjoints.

(d.2) For every $j \in \mathbb{Z}$ the family $\mathcal{D}^{j}$ covers almost all $X$ in the sense that $\mu(X-$ $\left.\bigcup_{Q \in \mathcal{D}^{j}} Q\right)=0$

(d.3) If $Q \in \mathcal{D}^{j}$ and $i<j$, then there exists a unique $\tilde{Q} \in \mathcal{D}^{i}$ such that $Q \subseteq \tilde{Q}$.

(d.4) If $Q \in \mathcal{D}^{j}$ and $\tilde{Q} \in \mathcal{D}^{i}$ with $i \leq j$, then either $Q \subseteq \tilde{Q}$ or $Q \cap \tilde{Q}=\emptyset$.

(d.5) There exists a positive integer $N$ depending only on $A$ such that for every $j \in \mathbb{Z}$ and all $Q \in \mathcal{D}^{j}$ the inequalities $1 \leq \#\left(\left\{Q^{\prime} \in \mathcal{D}^{j+1}: Q^{\prime} \subseteq Q\right\}\right) \leq N$ hold.

(d.6) There exist two constants $a_{1}$ and $a_{2}$ such that for each $Q \in \mathcal{D}^{j}$ we have that $r_{i}(Q) \geq a_{1} \delta^{j}$ and $r_{o}(Q) \leq a_{2} \delta^{j}$ and hence $\varepsilon(Q) \geq \frac{a_{1}}{a_{2}}$.

From property $(d .6)$ we are able to define center functions $\mathcal{P}^{j}$ assigning to each cube $Q \in \mathcal{D}$ points in $Q$ which could be considered geometric centers for $Q$ in a sense that we proceed to describe. For each $j \in \mathbb{Z}$ set $\mathcal{P}^{j}: \mathcal{D}^{j} \longrightarrow X$ given by $\mathcal{P}^{j}(Q)=x_{Q} \in Q$ such that $\frac{r_{i}(Q)}{2} \leq d\left(x_{Q}, X \backslash Q\right)$. Hence $B\left(x_{Q}, \frac{a_{1}}{2} \delta^{j}\right) \subseteq Q \subseteq$ $B\left(x_{Q}, 3 a_{2} \delta^{j}\right)$. Let us observe that this center function is not unique. In fact, even in the Euclidean setting it is possible to choose among several possible $x_{Q}$ for a given $Q$.

The following proposition contains the main properties of center functions. 
Proposition 2.2. Let $(X, d, \mu)$ be a metric space of homogeneous type and let $\mathcal{D}$ be a dyadic system in $\mathfrak{D}(\delta)$. Let $\mathcal{P}^{j}$ be a center function for each integer $j$. Then

(1) for each $j \in \mathbb{Z}$ we have that the set $\mathcal{P}^{j}\left(\mathcal{D}^{j}\right)$ is $\frac{a_{1}}{2} \delta^{j}$-disperse;

(2) the set $\mathcal{P}=\bigcup_{j \in \mathbb{Z}} \mathcal{P}^{j}\left(\mathcal{D}^{j}\right)$ is dense in $X$.

Proof. First we prove (1). Let $j \in \mathbb{Z}$ and $x$ and $y$ be two different points in $\mathcal{P}^{j}\left(\mathcal{D}^{j}\right)$. Let $Q_{1}=\left(\mathcal{P}^{j}\right)^{-1}(x)$ and $Q_{2}=\left(\mathcal{P}^{j}\right)^{-1}(y)$. Notice that $Q_{1}$ and $Q_{2}$ belong to $\mathcal{D}^{j}$ and that $\mathcal{P}_{j}$ is one to one. Thus, from (d.1), $Q_{1} \cap Q_{2}=\emptyset$. Suppose that $d(x, y)<\frac{a_{1}}{2} \delta^{j}$. Then $y \in B\left(x, \frac{a_{1}}{2} \delta^{j}\right)$ and therefore $y \in Q_{1}$. A contradiction. Hence $d(x, y) \geq \frac{a_{1}}{2} \delta^{j}$ and $\mathcal{P}_{j}\left(\mathcal{D}^{j}\right)$ is $\frac{a_{1}}{2} \delta^{j}$-disperse.

To prove (2), we take $x \in X$ and $\varepsilon>0$. Fix $j \in \mathbb{Z}$ such that $a_{2} \delta^{j}<\varepsilon$. From (d.2) it follows that $\bigcup_{Q \in \mathcal{D}^{j}} Q$ is dense in $X$. Hence, we may assume that $x \in \bigcup_{Q \in \mathcal{D}^{j}} Q$. Let $Q$ be the unique dyadic cube in $\mathcal{D}^{j}$ such that $x \in Q$. Then $x \in B\left(\mathcal{P}^{j}(Q), 3 a_{2} \delta^{j}\right)$ and therefore $d\left(x, \mathcal{P}^{j}(Q)\right) \leq d\left(\mathcal{P}^{j}(Q), X \backslash Q\right) \leq 3 a_{2} \delta^{j}<3 \varepsilon$.

It is easy to give examples of dyadic systems $\mathcal{D}([4])$ such that in some regions or at some scales the space may keep dividing in pieces that become smaller and smaller and such that at some regions, refining scales does not involve an actual refinement of the space. We are interested in the identification of those scales and places of partition which shall give rise to the Haar functions. This induces the definition of a subfamily of $\mathcal{D}$ containing all dyadic cubes in $\mathcal{D}$ with non-trivial offspring.

Definition 2.3. The subfamily $\tilde{\mathcal{D}}$ of a $\mathcal{D}$ in $\mathfrak{D}(\delta)$. For each $\mathcal{D}$ in $\mathfrak{D}(\delta)$ and for each $j \in \mathbb{Z}$ we considered the families

$$
\tilde{\mathcal{D}}^{j}=\left\{Q \in \mathcal{D}^{j}: \#\left(\left\{Q^{\prime} \in \mathcal{D}^{j+1}: Q^{\prime} \subseteq Q\right\}\right)>1\right\} .
$$

We define

$$
\tilde{\mathcal{D}}=\bigcup_{j \in \mathbb{Z}} \tilde{\mathcal{D}}^{j} .
$$

Properties (d.1) to (d.6) allow us to obtain the following result.

Proposition 2.4. Let $(X, d, \mu)$ be a metric space of homogeneous type and let $\mathcal{D}$ be in $\mathfrak{D}(\delta)$. Then

(d.7) The families $\tilde{\mathcal{D}}^{j}, j \in \mathbb{Z}$ are pairwise disjoints.

(d.8) The function $\mathcal{J}: \tilde{\mathcal{D}} \longrightarrow \mathbb{Z}$ given by $Q \mapsto \mathcal{J}(Q)$ if $Q \in \tilde{\mathcal{D}}^{\mathcal{J}(Q)}$ is well defined.

Proof. First notice that $(d .8)$ is an easy consequence of (d.7). For see (d.7) notice that given a cube $Q \in \tilde{\mathcal{D}}^{j}$, from (d.1) we have that $Q \notin \mathcal{D}^{j+1}$. Moreover, $Q \notin \mathcal{D}^{j+n}$ for any positive integer $n$ and hence $Q \notin \tilde{\mathcal{D}}^{j+n}$.

In the sequel two dyadic families $\mathcal{D}_{1}=\bigcup_{j \in \mathbb{Z}} \mathcal{D}_{1}^{j}$ and $\mathcal{D}_{2}=\bigcup_{j \in \mathbb{Z}} \mathcal{D}_{2}^{j}$ in $\mathfrak{D}(\delta)$ on $X$ are given. We shall denote by $Q$ the elements of $\mathcal{D}_{1}$, by $R$ those in $\mathcal{D}_{2}$, by $\mathcal{J}_{i}$ the function in (d.8) for $\mathcal{D}_{i}, i=1,2$ and by $\mathcal{P}_{i}^{j}$ a center function for $\mathcal{D}_{i}, i=1,2, j \in \mathbb{Z}$. It is easy to give examples of dyadic systems such that no wavelet occurs for some levels of resolution. Therefore, the conditions for the new notion of equivalence over the families $\mathcal{D}_{1}$ and $\mathcal{D}_{2}$ are given on the subfamilies $\tilde{\mathcal{D}}_{1}$ and $\tilde{\mathcal{D}}_{2}$. In [4] the authors introduce a notion of equivalence of two dyadic systems. It will became clear why 
this notion is not enough in order to reflect the rigidity of the dyadic setting. The right notion now needs to take more care about the distance of the related dyadic sets. With this idea in mind we make the following definition.

Definition 2.5. Equivalent dyadic families. We say that the two dyadic families $\mathcal{D}_{1}$ and $\mathcal{D}_{2}$ in $\mathfrak{D}(\delta)$ are equivalent, briefly $\mathcal{D}_{1} \approx \mathcal{D}_{2}$, if there exist a positive integer $n$ and a relation $\mathfrak{R} \subseteq \tilde{\mathcal{D}}_{1} \times \tilde{\mathcal{D}}_{2}$ such that

(i) for each $Q \in \tilde{\mathcal{D}}_{1}$ there exists $R \in \tilde{\mathcal{D}}_{2}$ such that $(Q, R) \in \mathfrak{R}$;

(ii) for each $R \in \tilde{\mathcal{D}}_{2}$ there exists $Q \in \tilde{\mathcal{D}}_{1}$ such that $(Q, R) \in \mathfrak{R}$;

(iii) for all $(Q, R) \in \mathfrak{R}$ we have $\left|\mathcal{J}_{1}(Q)-\mathcal{J}_{2}(R)\right| \leq n$;

(iv) for all $(Q, R) \in \mathfrak{R}$ there exists two dyadic cubes $Q^{\prime} \in \mathcal{D}_{1}^{\mathcal{J}_{1}(Q)+n}$ and $R^{\prime} \in$ $\mathcal{D}_{2}^{\mathcal{J}_{2}(R)+n}$ such that $Q^{\prime} \cup R^{\prime} \subseteq Q \cap R$.

Let us start by showing that the first non trivial example for this notion of equivalence of dyadic systems is provided by the well know Christ's dyadic families on a space of homogeneous type, when they are built on the same basic nets of points.

Let us briefly recall the construction given in [9]. Let $\delta>0$ be given. For each $j \in \mathbb{Z}$ a finite or countable sequence $\mathcal{N}_{j}=\left\{x_{k}^{j}: k \in \mathcal{K}_{j}\right\}$ which is maximal with the property $d\left(x_{k}^{j}, x_{l}^{j}\right) \geq \delta^{j}, k \neq j$ in $\mathcal{K}_{j}$, is given. Here $\mathcal{K}_{j}$ is an initial interval in $\mathbb{Z}^{+}$which may coincide with $\mathbb{Z}^{+}$. Set $\mathcal{A}=\left\{(j, k): j \in \mathbb{Z}\right.$ and $\left.k \in K_{j}\right\}$. The first step in Christ's construction is to introduce tree structures on the index set $\mathcal{A}$ that are closely related to the metric structure on $X$. In other words, M.Christ define a partial orders $\preceq$ on $\mathcal{A}$ satisfying some tree properties controlled by the distance. The second step in [9] is the construction of a dyadic family, $\mathcal{D}$, based on such partial orders $\preceq$ defined on $\mathcal{A}$. For a given sequence $\mathcal{N}_{j}, \delta>0$, we shall say that such order belongs to the class $\mathcal{C}$, briefly, $\preceq \in \mathcal{C}$.

In the definition of partial order $\preceq$ the choice of ancestors in the tree is not unique at all. This diversity translates into a corresponding diversity of families $\mathcal{D}$. On the other hand there is some rigidity which is reflected by the following property: For each $(j, k) \in \mathcal{A}$ there exists al least one $u \in K_{j+1}$ such that $(j+1, u) \preceq(j, k)$ for every order $\preceq \in \mathcal{C}$ (see [4]).

For a given $\preceq \in \mathcal{C}$, the Christ dyadic cube at the level $j$ located at $k \in K_{j}$ is defined by

$$
Q_{k}^{j}=\bigcup_{(i, l) \preceq(j, k)} B\left(x_{l}^{i}, a \delta^{i}\right),
$$

which for some small values of the positive constant $a$ satisfy $(d .1)$ to $(d .8)$. The set $Q_{k}^{j}$ shall be called the dyadic cube associated to $x_{k}^{j} \in \mathcal{N}_{j}$. The family $\mathcal{D}_{\preceq}$ of all those $Q_{k}^{j}$ shall be called the Christ cubes associated to the family $\left\{\mathcal{N}_{j}\right.$ : $j \in \mathbb{Z}$ \} of nets and the order $\preceq$. Notice that different orders satisfying all the desired properties produce different shapes for the sets $Q_{k}^{j}$ corresponding to the level-position parameters $(j, k) \in \mathcal{A}$.

Given $\mathcal{D}_{i}=\mathcal{D}_{\preceq_{i}}$, where $\preceq_{i} \in \mathcal{C}, i=1,2$, we shall denote by $\tilde{\mathcal{D}}_{i}$ the family in $(d .7)$ for $\mathcal{D}_{i}$, by $Q$ the elements $Q_{k}^{j}$ of $\mathcal{D}_{1}$, by $R$ those cubes $R_{k}^{j}$ in $\mathcal{D}_{2}$, and by $\mathcal{J}_{i}$ the function in $(d .8)$ for $\mathcal{D}_{i}$.

Proposition 2.6. Let $\preceq_{1}$ and $\preceq_{2}$ be two partial orders in $\mathcal{C}$ with $0<\delta<1 / 2$. Then $\mathcal{D}_{1} \approx \mathcal{D}_{2}$, where $\mathcal{D}_{i}=\mathcal{D}_{\unlhd_{i}}, i=1,2$. 
Proof. We shall use the notation $Q_{u}^{j} \simeq R_{k}^{j}$ if $B\left(z, a \delta^{j+1}\right) \subseteq Q_{u}^{j} \cap R_{k}^{j}$ for some $z \in X$, where $a$ is the real number in (2.2) and $j \in \mathbb{Z}$. Let us first notice that

(1) for every $Q_{k}^{j} \in \tilde{\mathcal{D}}_{1}$ there exist $R_{u}^{j} \in \tilde{\mathcal{D}}_{2}$ such that $R_{u}^{j} \simeq Q_{k}^{j}$;

(2) for every $R_{k}^{j} \in \tilde{\mathcal{D}}_{2}$ there exist $Q_{u}^{j} \in \tilde{\mathcal{D}}_{1}$ such that $Q_{u}^{j} \simeq R_{k}^{j}$.

In fact, from Lemma 5.2 in [4] we have that for each $Q_{k}^{j} \in \tilde{\mathcal{D}}_{1}$ there exists $R_{u}^{j} \in \tilde{\mathcal{D}}_{2}$ and $(j+1, s) \in \mathcal{A}$ such that $(j+1, s) \preceq_{1}(j, k)$ and $(j+1, s) \preceq_{2}(j, u)$. Then, clearly from the definition of Christ's dyadic cubes (2.2), we obtain (1) with $z=x_{s}^{j+1}$. Analogously we prove (2).

The proof of the proposition is now an immediate consequence of (1) and (2). In fact, let $\mathfrak{R}$ be the subset of $\tilde{\mathcal{D}}_{1} \times \tilde{\mathcal{D}}_{2}$ defined as

$$
\mathfrak{R}=\left(\bigcup_{Q_{k}^{j} \in \tilde{\mathcal{D}}_{1}} \bigcup_{R_{u}^{j}: R_{u}^{j} \simeq Q_{k}^{j}}\left(Q_{k}^{j}, R_{u}^{j}\right)\right) \bigcup\left(\bigcup_{R_{k}^{j} \in \tilde{\mathcal{D}}_{2}} \bigcup_{Q_{u}^{j}: Q_{u}^{j} \simeq R_{k}^{j}}\left(Q_{u}^{j}, R_{k}^{j}\right)\right) .
$$

Clearly $\mathfrak{R}$ satisfies (i) and (ii) in Definition 2.5.

Now we show that (iv) also holds. Let $\left(Q_{k}^{j}, R_{u}^{j}\right) \in \mathfrak{R}$ and $(j+1, s) \in \mathcal{A}$ such that $B\left(x_{s}^{j+1}, a \delta^{j+1}\right) \subseteq Q_{k}^{j} \cap R_{u}^{j}$. Since $\left\{x_{l}^{m}: m \in \mathbb{Z}, l \in \mathcal{K}_{m}\right\}$ is dense in $X$, there exist two positive integers $l_{0}$ and $n$ such that $d\left(x_{l_{0}}^{j+1+n}, x_{s}^{j+1}\right)<3 a_{2} \delta^{j+1+n}<$ $\frac{a \delta^{j+1}}{2}$, where $a_{2}$ is the constant in (d.6) for $\mathcal{D}_{1}$ and $\mathcal{D}_{2}$. Then $Q_{l_{0}}^{j+1+n} \cup R_{l_{0}}^{j+1+n} \subseteq$ $B\left(x_{s}^{j+1}, a \delta^{j+1}\right) \subseteq Q_{k}^{j} \cap R_{u}^{j}$. In fact, for each $z \in Q_{l_{0}}^{j+1+n} \cup R_{l_{0}}^{j+1+n}$ we have

$$
\begin{aligned}
d\left(z, x_{s}^{j+1}\right) & \leq d\left(z, x_{l_{0}}^{j+1+n}\right)+d\left(x_{l_{0}}^{j+1+n}, x_{s}^{j+1}\right) \\
& \leq 3 a_{2} \delta^{j+1+n}<a \delta^{j+1} .
\end{aligned}
$$

Hence (iv) in Definition 2.5 holds with that $\mathrm{n}$. Condition (iii) is trivial and therefore $\mathcal{D}_{1} \approx \mathcal{D}_{2}$.

Associated to a dyadic system $\mathcal{D}$ in $\mathfrak{D}(\delta)$ it is possible to define the dyadic Hardy-Littlewood maximal operator

$$
M_{\mathcal{D}} f(x)=\sup _{Q} \frac{1}{\mu(Q)} \int_{Q}|f(y)| d \mu(y),
$$

where the supremum is taken over the family of dyadic cubes $Q$ in $\mathcal{D}$ containing $x$. Since $E=\cup_{Q \in \mathcal{D}} \partial(Q)$ has measure zero we may think that $M_{\mid \text {mathcalD }} f(x)$ is defined by zero when $x \in E$.

The reason for the restriction in the generality of the relation $\mathfrak{R}$ giving the equivalence of $\mathcal{D}_{1}$ and $\mathcal{D}_{2}$, which is contained in (iv) of Definition 2.5 is reflected in the next result which shall be crucial.

Theorem 2.7. Let $\mathcal{D}_{i}, i=1,2$ be two dyadic systems in $\mathfrak{D}(\delta)$ such that $\mathcal{D}_{1} \approx \mathcal{D}_{2}$. Then there exists a positive constant $C$ such that for every $(Q, R) \in \mathfrak{R}$ we have

(a) $\chi_{Q}(x) \leq C M_{\mathcal{D}_{1}}\left(\chi_{R}\right)(x)$ and

(b) $\chi_{R}(x) \leq C M_{\mathcal{D}_{2}}\left(\chi_{Q}\right)(x)$,

for all $x \in X$. 
Proof. By symmetry we shall only prove $(a)$. Since $(Q, R) \in \mathfrak{R}$, from (iv) there exists $Q^{\prime} \in \mathcal{D}_{1}^{\mathcal{J}_{1}(Q)+n}$ such that $Q^{\prime} \subseteq Q \cap R$. Thus, from the doubling property we obtain that $\mu\left(Q^{\prime}\right) \leq \mu(Q) \leq C \mu\left(Q^{\prime}\right)$. Hence, for all $x \in Q$

$$
\begin{aligned}
M_{\mathcal{D}_{1}}\left(\chi_{R}\right)(x) & =\sup _{\tilde{Q} \in \mathcal{D}_{1}, x \in \tilde{Q}} \frac{1}{\mu(\tilde{Q})} \int_{\tilde{Q}} \chi_{R} d \mu \\
& \geq \frac{\mu(Q \cap R)}{\mu(Q)} \geq \frac{\mu\left(Q^{\prime}\right)}{\mu(Q)} \geq \frac{1}{C} .
\end{aligned}
$$

\section{Equivalence of HaAr systems}

In this section we shall study the equivalence of Haar systems associated to equivalents dyadic families in the sense of Definition 2.5. Given $\mathcal{D}$ a dyadic system, in the sequel we shall make use of the following notation. Let $\mathcal{L}$ be the function defined on $\tilde{\mathcal{D}}$ taking values in the family of subsets of $\mathcal{D}$, given by $\mathcal{L}(Q)=\left\{Q^{\prime} \in\right.$ $\left.\mathcal{D}^{j+1}: Q^{\prime} \subseteq Q\right\}$ for $Q \in \tilde{\mathcal{D}}^{j}$ and $j \in \mathbb{Z}$. We shall say that the cube $Q \in \tilde{\mathcal{D}}$ is the first-ancestor of $Q^{\prime}$ if $Q^{\prime} \in \mathcal{L}(Q)$. Notice that from $(d .5), 1<\#(\mathcal{L}(Q)) \leq N$.

Now we define what we mean by a Haar system associated to a dyadic family.

Definition 3.1. Haar system associated to $\mathcal{D} \in \mathfrak{D}(\delta)$. Let $\mathcal{D}$ be a dyadic family on $(X, d, \mu)$ such that $\mathcal{D} \in \mathfrak{D}(\delta)$. A system $\mathcal{H}$ of Borel measurable simple real functions $h$ on $X$ is a Haar system associated to $\mathcal{D}$ if it satisfies

(h.1) For each $h \in \mathcal{H}$ there exists a unique $j \in \mathbb{Z}$ and a cube $Q=Q(h) \in \tilde{\mathcal{D}}^{j}$ such that $\{x \in X: h(x) \neq 0\}=\bigcup_{Q^{\prime} \in \mathcal{L}(Q)} Q^{\prime}$, and this property does not hold for any cube in $\mathcal{D}^{j+1}$. Moreover, each function $h$ is constant in each cube $Q^{\prime} \in \mathcal{L}(Q(h))$.

(h.2) For every $Q \in \tilde{\mathcal{D}}$ there exist exactly $M_{Q}=\#(\mathcal{L}(Q))-1 \geq 1$ functions $h \in \mathcal{H}$ such that (h.1) holds. We shall write $\mathcal{H}_{Q}$ to denote the set of all these functions $h$.

(h.3) For each $h \in \mathcal{H}$ we have that $\int_{X} h d \mu=0$.

(h.4) For each $Q \in \tilde{\mathcal{D}}$ let $V_{Q}$ denote the vector space of all functions on $Q$ which are constant on each $Q^{\prime} \in \mathcal{L}(Q)$. Then the system $\left\{\frac{\chi_{Q}}{(\mu(Q))^{1 / 2}}\right\} \cup \mathcal{H}_{Q}$ is an orthonormal basis for $V_{Q}$.

(h.5) There exists a positive constant $C$ such that the inequality $|h(x)| \leq C|h(y)|$ holds for almost every $x$ and $y$ in $Q(h)$ and every $h \in \mathcal{H}$.

Notice that our present definition of Haar type systems associated to $\mathcal{D}$ is more restrictive than the one considered in [4]. In fact, there a Haar wavelet may vanish in one or several of the offspring of its essential support. Now, property (h.5) leads us to a situation which looks more similar to the classical Euclidean case, where the absolute value of the basic functions is one. It is not difficult to see that most of the orthonormal basis of $V_{Q}$ containing $\frac{\chi_{Q}}{(\mu(Q))^{1 / 2}}$ satisfy also (h.5), actually (h.5) is a generic property of the Haar systems.

The following result will be crucial for the proof of our main result in Section 3 
Lemma 3.2. Let $\mathcal{H}$ be a Haar system associated to $\mathcal{D}$. Then there exist two positive constants $c_{1}$ and $c_{2}$ such that the inequalities

$$
\frac{c_{1}}{\sqrt{\mu(Q(h))}} \leq|h(x)| \leq \frac{c_{2}}{\sqrt{\mu(Q(h))}},
$$

hold for every $h \in \mathcal{H}$ and for almost $x \in Q(h)$.

Proof. Let $h$ be a function in $\mathcal{H}$. From (h.4) and (h.1) we obtain that there exists a finite family of scalars $\left\{a_{Q^{\prime}}: Q^{\prime} \in \mathcal{L}(Q(h))\right\}$ such that $h(x)=\sum_{Q^{\prime} \in \mathcal{L}(Q(h))} a_{Q^{\prime}} \chi_{Q^{\prime}}$ and

$$
\|h\|_{L^{2}(X, \mu)}^{2}=\sum_{Q^{\prime} \in \mathcal{L}(Q(h))}\left|a_{Q^{\prime}}\right|^{2} \mu\left(Q^{\prime}\right)=1 .
$$

Thus, since $\left|a_{\tilde{Q}}\right|^{2} \mu(\tilde{Q}) \leq \sum_{Q^{\prime} \in \mathcal{L}(Q(h))}\left|a_{Q^{\prime}}\right|^{2} \mu\left(Q^{\prime}\right)$ for all $\tilde{Q} \in \mathcal{L}(Q(h))$ we obtain, from the doubling property, the upper bound.

Let $\tilde{Q} \in \mathcal{L}(Q(h))$ be fixed. From (h.5), we have that $\left|a_{Q^{\prime}}\right| \leq C\left|a_{\tilde{Q}}\right|$ for all $Q^{\prime} \in \mathcal{L}(Q(h))$. Then, from (3.1) we obtain

$$
1 \leq C^{2} \mu(Q(h))\left|a_{\tilde{Q}}\right|^{2}
$$

where $C$ is given by (h.5).

Therefore the lower bound holds with $c_{1}=\frac{1}{G \sqrt{\mu(Q(h)))}}$.

Let $\mathcal{H}_{1}=\{h\}$ and $\mathcal{H}_{2}=\{\psi\}$ be two Haar systems associated to $\mathcal{D}_{1}$ and $\mathcal{D}_{2}$ respectively, with $\mathcal{D}_{1} \approx \mathcal{D}_{2}$. Let $Q(h)$ and $R(\psi)$ be the dyadic cubes in (h.1) for $h \in \mathcal{H}_{1}$ and $\psi \in \mathcal{H}_{2}$ respectively. We shall say that $\Psi$ is a selection function in $\mathcal{H}_{2}$ associated to $\mathfrak{R}$ if $\Psi: \mathcal{H}_{1} \longrightarrow \mathcal{H}_{2}$ and for every $h \in \mathcal{H}_{1}$ we have that $(Q(h), R(\Psi(h))) \in \mathfrak{R}$. Notice that from the properties of $\mathfrak{R}$ all these selection functions are connecting wavelets in one system to wavelets in the other with similar supports in scale and intersecting supports. We shall denote by $\mathcal{S}_{1,2}$ the set of all such selection functions. Symmetrically we say that a function $\mathfrak{h}: \mathcal{H}_{2} \longrightarrow \mathcal{H}_{1}$ is a selection function in $\mathcal{H}_{1}$ associated to $\mathfrak{R}^{-1}$ if $(Q(\mathfrak{h}(\psi)), R(\psi)) \in \mathfrak{R}$. With $\mathcal{S}_{2,1}$ we denote the set of all such selection functions.

In order to state the main result of this section, we start by some basic notation and definitions from dyadic harmonic analysis.

When a dyadic system $\mathcal{D}$ is given, we can define, as usual, the dyadic Muckenhoupt weight functions associated to $\mathcal{D}$. In fact, a non-negative, measurable and locally integrable function $w$ defined on the space of homogeneous type $(X, d, \mu)$, is said to be a Muckenhoupt dyadic weight of class $A_{p}^{\mathcal{D}}, 1<p<\infty$, if the inequality

$$
\left(\frac{1}{\mu(Q)} \int_{Q} w(x) d \mu(x)\right)\left(\frac{1}{\mu(Q)} \int_{Q} w(x)^{\frac{-1}{p-1}} d \mu(x)\right)^{p-1} \leq C
$$

holds for some constant $C$ and every dyadic set $Q \in \mathcal{D}$.

For $p=1$ we say that $w \in A_{1}^{\mathcal{D}}$ if there is a constant $C$ such the inequality

$$
\frac{w(Q)}{\mu(Q)} \leq C w(x)
$$

holds for almost all point $x \in Q$ and for every dyadic cube $Q \in \mathcal{D}$. 
We shall use the notation $\|f\|_{p, w}$ for the norm $\left(\int_{X}|f|^{p} w d \mu\right)^{1 / p}$ in $L_{w}^{p}(X, \mu)$, $1 \leq p<\infty$ and $w$ a non-negative function on $X$.

Theorem 3.3. Let $\mathcal{D}_{1}$ and $\mathcal{D}_{2}$ be two dyadic families on $(X, d, \mu)$ such that $\mathcal{D}_{1} \approx$ $\mathcal{D}_{2}$. Let $\mathcal{H}_{1}$ and $\mathcal{H}_{2}$ two Haar systems associated to $\mathcal{D}_{1}$ and $\mathcal{D}_{2}$ respectively. Then, for each $\mathfrak{R} \subseteq \tilde{\mathcal{D}}_{1} \times \tilde{\mathcal{D}}_{2}$ as in Definition 2.5, for every choice of $w_{i} \in A_{p}^{\mathcal{D}_{i}}, i=1,2$, there exist two positive constants $C_{i}, i=1,2$, depending of the $A_{p}^{\mathcal{D}_{i}}$ constant of $w_{i}$ such that for every finite subsets $F$ of $\mathcal{H}_{1}$ and $G$ of $\mathcal{H}_{2}$, every choice of sequences $\left(\lambda_{h}: h \in \mathcal{H}_{1}\right)$ and $\left(\nu_{\psi}: \psi \in \mathcal{H}_{2}\right)$ of real numbers and every couple of selection functions $\Psi \in \mathcal{S}_{1,2}$ and $\mathfrak{h} \in \mathcal{S}_{2,1}$ associated to $\mathfrak{R}$ we have the inequalities

$$
\left\|\sum_{h \in F} \lambda_{h} h\right\|_{p, w_{1}} \leq C_{1}\left\|\sum_{h \in F} \lambda_{h} \Psi(h)\right\|_{p, w_{1}}
$$

and

$$
\left\|\sum_{\psi \in G} \nu_{\psi} \psi\right\|_{p, w_{2}} \leq C_{2}\left\|\sum_{\psi \in G} \nu_{\psi} \mathfrak{h}(\psi)\right\|_{p, w_{2}} .
$$

Notice that if there exists $\Psi \in \mathcal{S}_{1,2}$ such that $\Psi$ is one to one and onto, using (3.3) with $\mathfrak{h}=\Psi^{-1}$ we obtain the result contained in the next statement.

Corollary 3.4. Let $\mathcal{D}_{1}, \mathcal{D}_{2}, \mathcal{H}_{1}$ and $\mathcal{H}_{2}$ be as in Theorem 3.3. Assume that in $\mathcal{S}_{1,2}$ there exists a one to one and onto selection function $\Psi$. Then, for each $w \in A_{p}^{\mathcal{D}_{1}} \cap A_{p}^{\mathcal{D}_{2}}, 1<p<\infty$, there exist two positive constants $C_{1}$ and $C_{2}$ such that

$$
C_{1}\left\|\sum_{h \in F} \lambda_{h} h\right\|_{p, w} \leq\left\|\sum_{h \in F} \lambda_{h} \Psi(h)\right\|_{p, w} \leq C_{2}\left\|\sum_{h \in F} \lambda_{h} h\right\|_{p, w}
$$

hold for every finite subset $F$ of $\mathcal{H}_{1}$ and every sequence $\left(\lambda_{h}: h \in \mathcal{H}_{1}\right)$ of real numbers.

A few remarks are in order. First notice that, since the standard $A_{p}$ Muckenhoupt condition over the family of $d$-balls in $X$ is stronger than any $A_{p}^{\mathcal{D}}$, then $A_{p}^{\mathcal{D}_{1}} \cap A_{p}^{\mathcal{D}_{2}} \supseteq A_{p}$. Hence $A_{p}^{\mathcal{D}_{1}} \cap A_{p}^{\mathcal{D}_{2}}$ is non-trivial and inequalities (3.4) hold for standard $A_{p}$ weights.

Second, since several different Haar systems satisfying (h.1) to (h.5) can be built on the same underlying dyadic structure $\mathcal{D}$, inequalities (3.4) hold for such Haar systems with $w \in A_{p}^{\mathcal{D}}$, which is usually larger than $A_{p}$.

Third, it could also happen that two different dyadic systems share the same dyadic weights. In other words, it is possible, and actualy not hard, to produce two dyadic systems $\mathcal{D}_{1}$ and $\mathcal{D}_{2}$ in $\mathbb{R}$ with $\mathcal{D}_{1} \neq \mathcal{D}_{2}$ but $A_{p}^{\mathcal{D}_{1}}=A_{p}^{\mathcal{D}_{2}} \neq A_{p}$. In fact, even in the general setting, we only need the following property: there exists a positive constant $C$ such that

(a) for every $Q \in \mathcal{D}_{1}$ there exists $R \in \mathcal{D}_{2}$ such that $Q \subseteq R$ and $\mu(Q) \leq C \mu(R)$;

(b) for every $R \in \mathcal{D}_{2}$ there exists $Q \in \mathcal{D}_{1}$ such that $R \subseteq Q$ and $\mu(R) \leq C \mu(Q)$.

The proof of Theorem 3.3 is relies on the two following basic tools. The first one is a characterization of dyadic weighted Lebesgue spaces via Haar coefficients. The second is a dyadic version of Fefferman-Stein inequality. 
Theorem 3.5. Let $\mathcal{H}$ be any Haar system associated to $\mathcal{D} \in \mathfrak{D}(\delta)$. Let $w$ be a dyadic Muckenhoupt weight of class $A_{p}^{\mathcal{D}}, 1<p<\infty$. Then the system $\mathcal{H}$ is an unconditional basis of each $L_{w}^{p}(X, \mu), 1<p<\infty$. Moreover there exist two positive constants $C_{1}$ and $C_{2}$ depending of the $A_{p}^{\mathcal{D}}$ constant of $w$, such that the inequalities

$$
C_{1}\|f\|_{p, w} \leq\left\|\left(\sum_{h \in \mathcal{H}}|<f, h>|^{2}|h|^{2}\right)^{1 / 2}\right\|_{p, w} \leq C_{2}\|f\|_{p, w},
$$

hold for all $f \in L_{w}^{p}(X, \mu)$ with bounded support and vanishing integral.

Theorem 3.6. Let $\mathcal{D}$ be a dyadic system in $\mathfrak{D}(\delta)$. Let $w \in A_{p}^{\mathcal{D}}$ with $1<p<\infty$. Then there exists a constant $C$ depending of the $A_{p}^{\mathcal{D}}$ constant of $w$ and the geometric constants such that

$$
\left\|\left(\sum_{n \in \mathbb{N}}\left|M_{\mathcal{D}} f_{n}(.)\right|^{2}\right)^{1 / 2}\right\|_{p, w} \leq C\left\|\left(\sum_{n \in \mathbb{N}}\left|f_{n}(.)\right|^{2}\right)^{1 / 2}\right\|_{p, w}
$$

for every sequence of measurable functions $f_{n}$.

Theorem 3.5 can be proved as Theorem 9.1 in [3]. See [16] for previous related results. In section 4 we shall prove a more general version of Theorem 3.6.

Now, we prove the main result of this section.

Proof of Theorem 3.3. The chain of inequalities leading as to (3.2) and (3.3) are essentially the same as that in the proof of Theorem 3.5 in [4]. The main difference relies in the use of Lemma 3.2 and the fact that the maximal operator involved are $M_{\mathcal{D}_{1}}$ for (3.2) and $M_{\mathcal{D}_{2}}$ for (3.3). Let us briefly sketch the proof of (3.2).

First notice that, from Definition 2.5, there exists two positive constants $c$ and $C$ such that

$$
c \mu(R(\psi)) \leq \mu(Q(h)) \leq C \mu(R(\psi)),
$$

for every $h \in \mathcal{H}_{1}$ and every $\psi \in \mathcal{H}_{2}$ with $(Q(h), R(\psi)) \in \mathfrak{R}$.

Let $F$ be a finite subset of $\mathcal{H}_{1}, \Psi \in \mathcal{S}_{1,2}$ and let $\left(\lambda_{h}: h \in \mathcal{H}_{1}\right)$ be a sequence of scalars. From (3.5) and Lemma 3.2 we have that the inequalities

$$
\begin{aligned}
\sum_{h \in F} \lambda_{h}^{2}|h(x)|^{2} & \leq \sum_{h \in F} \lambda_{h}^{2} \frac{c_{2}^{2}}{\mu(Q(h))} \chi_{Q(h)}(x) \\
& \leq \frac{c_{2}^{2}}{c} \sum_{h \in F} \lambda_{h}^{2} \frac{1}{\mu(R(\Psi(h)))} \chi_{Q(h)}(x)
\end{aligned}
$$

and

$$
\sum_{h \in F} \lambda_{h}^{2} \frac{c_{1}^{2}}{\mu(R(\Psi(h)))} \chi_{R(\Psi(h))}(x) \leq \sum_{h \in F} \lambda_{h}^{2}|\Psi(h)(x)|^{2},
$$

hold for almost every $x \in X$. Applying Theorem 3.5 for the system $\mathcal{H}_{1}$, inequalities (3.6), Theorem 2.7, Theorem 3.6, inequality (3.7) and Theorem 3.5 for the system $\mathcal{H}_{2}$, we get 


$$
\begin{gathered}
\left\|\sum_{h \in F} \lambda_{h} h\right\|_{p, w_{1}} \leq C\left\|\left(\sum_{h \in F} \lambda_{h}^{2}|h|^{2}\right)^{1 / 2}\right\|_{p, w_{1}} \\
\leq C\left\|\left(\sum_{h \in F}\left[M_{\mathcal{D}_{1}}\left(\lambda_{h} \frac{1}{\sqrt{\mu(R(\Psi(h)))}} \chi_{R(\Psi(h))}\right)\right]^{2}\right)^{1 / 2}\right\|_{p, w_{1}} \\
\leq C\left\|\sum_{h \in F} \lambda_{h} \Psi(h)\right\|_{p, w_{1}},
\end{gathered}
$$

where the constant $c$ vary from one line to another but independently on the function $h$ in $F$.

If $\preceq_{1}$ and $\preceq_{2}$ are two partial orders in $\mathcal{C}$ as in Proposition 2.6 and $\mathcal{H}_{1}=\{h\}$ and $\mathcal{H}_{2}=\{\psi\}$ are two Haar systems associated with $\mathcal{D}_{1}$ and $\mathcal{D}_{2}$ respectively we obtain the conclusion of Theorem 3.3. Moreover, there exists a one to one and onto function $\Psi$ of $\mathcal{H}_{1}$ on $\mathcal{H}_{2}$ in $S_{1,2}$ (see [4]) and therefore we obtain the conclusion of Corollary 3.4 for the systems $\mathcal{H}_{1}$ and $\mathcal{H}_{2}$.

\section{FefFerman-Stein DyAdic Weighted inEQUALity For the DYADiC HARDY-LITTLEWOOD MAXIMAL OPERATOR}

Fefferman-Stein inequality for vector-valued maximal functions has became an important tool in several problems in harmonic analysis. As in the original paper by Fefferman and Stein ([10]), both, weak and strong type inequalities are proved by Andersen and John for weighted Lebesgue spaces in $\mathbb{R}^{n}([5])$. These results were proved in [11] in the setting of space of homogeneous type. A non weighted dyadic version in the Euclidean context is given in [17]. In this section we shall prove a weighted dyadic version on spaces of homogeneous type following the line of the proof in [5].

For a given sequence $\mathbf{f}=\left(f_{n}: n \in \mathbb{N}\right)$ of functions defined on $X$ we shall use the notation $\|\mathbf{f}(x)\|_{\ell^{r}}=\left(\sum_{n}\left|f_{n}(x)\right|^{r}\right)^{1 / r}$, for $1<r<\infty$. If $T$ is an operator on scalar functions we can extend $T$ to a vector valued operator by defining $T \mathbf{f}=\left(T f_{n}: n \in\right.$ $\mathbb{N})$.

Theorem 4.1. Let $(X, d, \mu)$ be a space of homogeneous type. Let $\mathcal{D}$ be a dyadic system in $\mathfrak{D}(\delta)$ and $1<r<\infty$. Then

(a) if $1 \leq p<\infty$, there exists a constant $C$ such that

$$
w\left(\left\{x \in X:\left\|M_{\mathcal{D}} \mathbf{f}(x)\right\|_{\ell^{r}}>\lambda\right\}\right) \leq C \frac{1}{\lambda^{p}} \int_{X}\|\mathbf{f}(x)\|_{\ell^{r}}^{p} w(x) d \mu(x)
$$

for every sequence of measurable functions $\mathbf{f}$ and every $\lambda>0$, if and only if $w \in A_{p}^{\mathcal{D}}$;

(b) if $1<p<\infty$, there exists a constant $C$ such that

$$
\int_{X}\left\|M_{\mathcal{D}} \mathbf{f}(x)\right\|_{\ell^{r}}^{p} w(x) d \mu(x) \leq C \int_{X}\|\mathbf{f}(x)\|_{\ell^{r}}^{p} w(x) d \mu(x)
$$


for every sequence of measurable functions $\mathbf{f}$, if and only if $w \in A_{p}^{\mathcal{D}}$.

The following theorem collects the properties of the dyadic Muckenhoupt weights on spaces of homogeneous type that we shall use in the sequel. Given a Borel positive measure $\nu$ on $X$ we shall say that $\nu$ is $\mathcal{D}$-doubling if there is a positive constant $C$ such that $\nu(\tilde{Q}) \leq C \nu(Q)$ for all $Q \in \mathcal{D}$, where $\tilde{Q}$ is the first-ancestor of $Q$.

Theorem 4.2. Let $1<p<\infty$ and $p^{\prime}$ such that $1 / p+1 / p^{\prime}=1$. The following statements hold:

(a) $w \in A_{p}^{\mathcal{D}}$ then $w(x)>0$ a.e.,

(b) if $w \in A_{p}^{\mathcal{D}}$ then $w d \mu$ is $\mathcal{D}$-doubling,

(c) $w \in A_{p}^{\mathcal{D}}$ then $w \in A_{q}^{\mathcal{D}}$ for every $q>p$,

(d) $w \in A_{p}^{\mathcal{D}}$ if and only if $w^{1-p^{\prime}} \in A_{p^{\prime}}^{\mathcal{D}}$,

(e) $w \in A_{p}^{\mathcal{D}}$ then $w \in A_{q}^{\mathcal{D}}$ for some $q<p$.

(f) $w \in A_{p}^{\mathcal{D}}$ is both necessary and sufficient for the weak and strong type $(p, p)$ inequality for the operator $M_{\mathcal{D}}$ with respect to the measure $w d \mu$,

(g) $w \in A_{1}^{\mathcal{D}}$ is both necessary and sufficient for the weak type $(1,1)$ inequality for the operator $M_{\mathcal{D}}$ with respect to the measure $w d \mu$.

The proof of Theorem 4.2 can be obtained as in the case of classical Muckenhoupt weights following the lines in [2] and [3] for the dyadic Muckenhoupt weights associated to Christ's dyadic cubes.

To prove Theorem 4.1 we shall use two classical tools. The first one is a CalderónZygmund decomposition of integrable functions associated to the system $\mathcal{D}$. We can obtain it in our general context of the dyadic system $\mathcal{D}$ in $\mathfrak{D}(\delta)$ following the lines in [3]. The second tool is interpolation.

The precise dyadic Calderón-Zygmund decomposition of integrable functions is contained in the following result.

Theorem 4.3. Let $(X, d, \mu)$ be a space of homogeneous and $\mathcal{D}$ be a dyadic system in $\mathfrak{D}(\delta)$. Let $f$ be a nonnegative integrable function defined on $X$. Let $\lambda>0$ be such that $\lambda \geq m_{X}(f)=\frac{1}{\mu(X)} \int_{X} f d \mu\left(m_{X}(f)=0\right.$ if $\left.\mu(X)=\infty\right)$. Then there exists a sequence $\mathcal{F}=\left\{Q_{i}\right\}_{i \in I}$ of dyadic cubes in $\mathcal{D}$ such that

(a) $Q_{i} \cap Q_{j}=\emptyset$ for $i \neq j$;

(b) $m_{Q_{i}}(f)=\frac{1}{\mu\left(Q_{i}\right)} \int_{Q_{i}} f d \mu>\lambda$ for every $i \in I$;

(c) $m_{\tilde{Q}}(f) \leq \lambda$ for every dyadic cube $\tilde{Q} \in \mathcal{D}$ such that $Q \subset \tilde{Q}, \tilde{Q} \neq Q$, for some $Q \in \mathcal{F}$

(d) $m_{Q^{\prime}}(f) \leq \lambda$ for every dyadic cube $Q^{\prime} \in \mathcal{D}$ such that $Q^{\prime} \cap\left(\bigcup_{Q \in \mathcal{F}} Q\right)=\emptyset$;

(e) $\Omega_{\lambda}:=\bigcup_{i \in I} Q_{i}=\left\{x \in X: M_{\mathcal{D}} f(x)>\lambda\right\}$;

(f) $|f(x)| \leq C \lambda$ for almost every $x \notin \Omega_{\lambda}$.

Now we state the two interpolation results that we shall use. We would like to point out that the geometric nature of the domain of the functions is irrelevant. For details concerning the results stated in this paragraph, see [6], [7] and [8], where the authors introduce the basic setting of vector valued operators and Lebesgue spaces with mixed norms. The mixed space $L^{p}\left(\ell^{r}\right)$ is the space of all sequences $\mathbf{f}=\left(f_{n}: n \in \mathbb{N}\right)$ for which $\int_{X}\|\mathbf{f}(x)\|_{\ell^{r}}^{p} d \mu(x)<\infty$, and we say that $\mathbf{f}$ belongs 
to $L_{w}^{p}\left(\ell^{r}\right)$ when $\int_{X}\|\mathbf{f}(x)\|_{\ell^{r}}^{p} w(x) d \mu(x)<\infty$ for some non-negative measurable $w$ defined on $X$.

We shall say that a scalar valued function is simple if it belongs to the linear span of the indicator functions of bounded measurable sets. Let $S$ be the class of all sequences $\mathbf{f}$ such that each $f_{n}$ is a simple function and $f_{n} \equiv 0$ for $n$ large enough. It is easy to show that $S$ is dense in each $L_{w}^{p}\left(\ell^{r}\right)(1 \leq p, r<\infty)$ when $w$ is a locally integrable non-negative function (see [7]).

Lemma 4.4. Let $w(x) \geq 0$ be locally integrable on $X, 1<r<\infty, 1 \leq p_{i}<\infty$ and suppose that $T$ is a sublinear operator defined on $S$ satisfying

$$
w\left(\left\{x \in X:\|T \mathbf{f}(x)\|_{\ell^{r}}>\alpha\right\}\right) \leq \frac{N_{i}^{p_{i}}}{\alpha^{p_{i}}} \int_{X}\|\mathbf{f}(x)\|_{\ell^{r}}^{p_{i}} w(x) d \mu(x)
$$

for some $N_{i}$, for $i=0,1$ and $\mathbf{f} \in S$. Then $T$ extends uniquely to a sublinear operator on $L_{w}^{p}\left(l^{r}\right)$ and there is a constant $N_{\theta}$ such that

$$
\left(\int_{X}\|T \mathbf{f}(x)\|_{\ell^{r}}^{p} w(x) d \mu(x)\right)^{1 / p} \leq N_{\theta}\left(\int_{X}\|\mathbf{f}(x)\|_{\ell^{r}}^{p} w(x) d \mu(x)\right)^{1 / p}
$$

where $\frac{1}{p}=(1-\theta) \frac{1}{p_{0}}+\theta \frac{1}{p_{1}}, 0 \leq \theta \leq 1$.

Lemma 4.5. Let $w(x) \geq 0$ be locally integrable on $X, 1<p<\infty, 1 \leq r_{i}<\infty$ and suppose that $T$ is a sublinear operator defined on $S$ satisfying

$$
\left(\int_{X}\|T \mathbf{f}(x)\|_{\ell^{r_{i}}}^{p} w(x) d \mu(x)\right)^{1 / p} \leq N_{i}\left(\int_{X}\|\mathbf{f}(x)\|_{\ell^{r_{i}}}^{p} w(x) d \mu(x)\right)^{1 / p}
$$

for some $N_{i}$, for $i=0,1$ and $\mathbf{f} \in S$. Then $T$ extends uniquely to a sublinear operator on $L_{w}^{p}\left(\ell^{r}\right)$ such that

$$
\left(\int_{X}\|T \mathbf{f}(x)\|_{\ell^{r}}^{p} w(x) d \mu(x)\right)^{1 / p} \leq N_{0}^{1-\theta} N_{1}^{\theta}\left(\int_{X}\|\mathbf{f}(x)\|_{\ell^{r}}^{p} w(x) d \mu(x)\right)^{1 / p}
$$

where $\frac{1}{r}=(1-\theta) \frac{1}{r_{0}}+\theta \frac{1}{r_{1}}, 0 \leq \theta \leq 1$.

Before proving Theorem 4.1 we shall first prove the following result that is an extension to spaces of homogeneous type of another central result due to Fefferman and Stein.

Proposition 4.6. Let $w$ be a nonnegative locally integrable function such that $w d \mu$ is $\mathcal{D}$-doubling. Then

(a) there exists $C>0$ such that the inequality

$$
w\left(\left\{x \in X: M_{\mathcal{D}} f(x)>\lambda\right\}\right) \leq \frac{C}{\lambda} \int_{X}|f(x)| M_{\mathcal{D}} w(x) d \mu(x)
$$

holds for every measurable function $f$ and every $\lambda>0$;

(b) given $1<p<\infty$, there exists a positive constant $C_{p}$ such that the inequality

$$
\int_{X}\left(M_{\mathcal{D}} f(x)\right)^{p} w(x) d \mu(x) \leq C_{p} \int_{X}|f(x)|^{p} M_{\mathcal{D}} w(x) d \mu(x)
$$

holds for every measurable function $f$. 
Proof. Notice that, except for the trivial case when $w \equiv 0$, since $M_{\mathcal{D}} w$ is positive at each point of $X$, we have that $\left\|M_{\mathcal{D}} f\right\|_{\infty, w} \leq\|f\|_{\infty, M_{\mathcal{D}} w}$. Hence, from the Marcinkiewicz interpolation theorem we only have to prove $(a)$.

Let $\lambda>0$ be given. Assume first that $\lambda \geq m_{X}(f)$. Then we can apply Theorem 4.3 to $f$ at the level $\lambda$ and we obtain a sequence $\mathcal{F}=\left\{Q_{i}\right\}_{i \in I}$ of disjoint dyadic cubes in $\mathcal{D}$ such that $m_{Q_{i}}(f)>\lambda$ for every $i \in I$ and $\Omega_{\lambda}=\bigcup_{i \in I} Q_{i}=\{x \in X$ : $\left.M_{\mathcal{D}} f(x)>\lambda\right\}$. Then, there exists a positive constant $C$ such that for each $Q_{i} \in \mathcal{F}$

$$
\int_{\tilde{Q}_{i}} w(x) d \mu(x) \leq \frac{C}{\lambda} \int_{Q_{i}}|f(y)| M_{\mathcal{D}} w(y) d \mu(y),
$$

where $\tilde{Q}_{i}$ is the first-ancestor of $Q_{i}$. In fact, since $w$ is $\mathcal{D}$-doubling, for each $Q_{i} \in \mathcal{F}$ we obtain

$$
\int_{\tilde{Q}_{i}} w d \mu \leq \frac{C}{\lambda} \int_{Q_{i}}|f|\left(\frac{1}{\mu\left(\tilde{Q}_{i}\right)} \int_{\tilde{Q}_{i}} w d \mu\right) d \mu \leq \frac{C}{\lambda} \int_{Q_{i}}|f| M_{\mathcal{D}} w d \mu .
$$

Hence

$$
w\left(\Omega_{\lambda}\right) \leq \sum_{i \in I} w\left(\tilde{Q}_{i}\right) \leq \sum_{i \in I} \frac{C}{\lambda} \int_{Q_{i}}|f| M_{\mathcal{D}} w d \mu \leq \frac{C}{\lambda} \int_{X}|f| M_{\mathcal{D}} w d \mu .
$$

Assume now that $0<\lambda<m_{X}(f)$. Hence $\mu(X)<\infty$ and therefore $X$ itself is a dyadic cube in $\mathcal{D}$. So, as in the proof of (4.1) we obtain that $\int_{X} w d \mu \leq$ $\frac{1}{\lambda} \int_{X}|f| M_{\mathcal{D}} w d \mu$. Therefore

$$
w\left(\left\{x \in X: M_{\mathcal{D}} f(x)>\lambda\right\}\right) \leq \int_{X} w(x) d \mu \leq \frac{1}{\lambda} \int_{X}|f| M_{\mathcal{D}} w d \mu .
$$

Proof of Theorem 4.1 Concerning the necessity of $A_{p}^{\mathcal{D}}$, there is nothing to show since $w \in A_{p}^{\mathcal{D}}$ is already necessary in the scalar-valued case achieved when $f_{n}(x)=$ $0, n=2,3, \ldots$.

To proof the converse let us notice that $(b)$ and hence $(a)$ for the case $p=r$ follows from Beppo-Levi Theorem and the boundedness in $L_{w}^{p}(X, d \mu)$ for $M_{\mathcal{D}} f$. Then we shall proof $(a)$ and $(b)$ for the case $p<r$. Finally a duality argument is used to show $(b)$ and hence $(a)$ for $r<p$.

We start proving $(a)$ for $1 \leq p<r$. Let $w \in A_{p}^{\mathcal{D}}$ and suppose first that $\mathbf{f} \in S$. The general case is obtained by density following standard arguments.

Take $\lambda>0$ and set $\psi(x)=\|\mathbf{f}(x)\|_{\ell^{r}}$. Since $f_{n} \equiv 0$ for $n$ large and each $f_{n}$ is a simple function with bounded support, it is clear that $\psi$ belongs to each $L_{w}^{p}(X, d \mu)$. Being $\lambda>0$ if the whole space $X$ is unbounded, or equivalently of infinite $\mu$-measure, we have that, $\lambda>m_{X}(\psi)$.

Since $\mu(X)=\infty$ is not the general situation for spaces of homogeneous type, in this point of the proof we have to take special care of the case when $X$ is bounded. Assume then that $0<\lambda<m_{X}(\psi)$. Suppose first that $p>1$. Since $w \in A_{p}^{\mathcal{D}}$ and $X$ itself is a dyadic cube we have, from Hölder inequality, that

$$
\int_{X} \psi d \mu \leq\left(\int_{X} \psi^{p} w d \mu\right)^{1 / p}\left(\int_{X} w^{-\frac{p^{\prime}}{p}} d \mu\right)^{\frac{p-1}{p}} \leq C\left(\int_{X} \psi^{p} w d \mu\right)^{1 / p} \frac{\mu(X)}{w(X)^{1 / p}} .
$$

Hence

$$
w(X) \leq \frac{C^{p}}{\lambda^{p}} \int_{X} \psi^{p} w d \mu
$$


If $p=1$,

$$
w(X)<\frac{1}{\lambda} \int_{X} \psi(y) \frac{w(X)}{\mu(X)} d \mu(y) \leq \frac{1}{\lambda} \int_{X} \psi(y) w(y) d \mu(y),
$$

where in the last inequality we have used that $w \in A_{1}^{\mathcal{D}}$ and that $X$ is a dyadic cube. Therefore the inequality in $(a)$ is proved if $0<\lambda<m_{X}(\psi)$, since $\{x \in X$ : $\left.\left\|M_{\mathcal{D}} \mathbf{f}(x)\right\|_{\ell^{r}}>\lambda\right\} \subseteq X$.

Hence we only have to deal with the case $\lambda \geq m_{X}(\psi)$. Let $\mathcal{F}=\left\{Q_{i}: i \in I\right\}$ be the family given by Theorem 4.3 applied to the function $\psi$ and to $\lambda$. Let $\Omega=\Omega_{\lambda}=\bigcup_{i \in I} Q_{i}$. We write each $f_{n}$ as $f_{n}=f_{n, 1}+f_{n, 2}$, where $f_{n, 1}=f_{n} \chi_{X \backslash \Omega}$ and $f_{n, 2}=f_{n} \chi_{\Omega}$. Thus, $\mathbf{f}=\mathbf{f}_{1}+\mathbf{f}_{2}$, where $\mathbf{f}_{1}=\left(f_{n, 1}: n \in \mathbb{N}\right)$. From the sublinearity of the Hardy-Littlewood dyadic maximal function and Minkowski inequality we get that

$$
\left\|M_{\mathcal{D}} \mathbf{f}(x)\right\|_{\ell^{r}} \leq\left\|M_{\mathcal{D}} \mathbf{f}_{1}(x)\right\|_{\ell^{r}}+\left\|M_{\mathcal{D}} \mathbf{f}_{2}(x)\right\|_{\ell^{r}}=I_{1}(x)+I_{2}(x) .
$$

Let us start proving the weak type inequality for $I_{1}(x)$. Since, from Theorem 4.3 $\psi(x)=\|\mathbf{f}(x)\|_{\ell^{r}} \leq C \lambda$ for almost every $x \notin \Omega$, we obtain for such $x$

$$
\left\|\mathbf{f}_{1}(x)\right\|_{\ell^{r}}^{r}=\left\|\mathbf{f}_{1}(x)\right\|_{\ell^{r}}^{r-p}\left\|\mathbf{f}_{1}(x)\right\|_{\ell^{r}}^{p} \leq C \lambda^{r-p}\left\|\mathbf{f}_{1}(x)\right\|_{\ell^{r}}^{p} .
$$

Therefore, from Theorem $4.2(f)$, we have

$$
\begin{aligned}
w\left(\left\{x \in X:\left\|M_{\mathcal{D}} \mathbf{f}_{1}(x)\right\|_{\ell^{r}}>\lambda / 2\right\}\right) & \leq \frac{2^{r}}{\lambda^{r}} \int_{X}\left\|M_{\mathcal{D}} \mathbf{f}_{1}(x)\right\|_{\ell^{r}}^{r} w(x) d \mu(x) \\
& \leq \frac{C 2^{r}}{\lambda^{r}} \int_{X}\left\|\mathbf{f}_{1}(x)\right\|_{\ell^{r}}^{r} w(x) d \mu(x) \\
& \leq \frac{C 2^{r}}{\lambda^{p}} \int_{X}\|\mathbf{f}(x)\|_{\ell^{r}}^{p} w(x) d \mu(x) .
\end{aligned}
$$

In order to obtain the result for $I_{2}(x)$, we define for each $n \in \mathbb{N}$ the function

$$
\tilde{f}_{n}(x)=\left\{\begin{array}{cc}
\frac{1}{\mu\left(Q_{i}\right)} \int_{Q_{i}}\left|f_{n}(y)\right| d \mu(y) & \text { if } x \in Q_{i}, \\
0 & \text { if } x \notin \Omega
\end{array}\right.
$$

and we shall prove that

$$
M_{\mathcal{D}} f_{n, 2}(x) \leq C M_{\mathcal{D}} \tilde{f}_{n}(x),
$$

for every $n \in \mathbb{N}$ and every $x \notin \Omega$. In fact, let $x \notin \Omega$ and let $Q \in \mathcal{D}$ be a dyadic cube containing $x$. Set $J=\left\{j: Q_{j} \in \mathcal{F}\right.$ and $\left.Q_{j} \cap Q \neq \emptyset\right\}$. Notice that, if $j \in J$, since $Q_{j}$ and $Q$ are dyadic cubes, then $Q_{j} \subseteq Q$ and from the definition of $f_{n, 2}$ we get

$$
\frac{1}{\mu(Q)} \int_{Q}\left|f_{n, 2}\right| d \mu=\frac{1}{\mu(Q)} \sum_{j \in J} \int_{Q_{j} \cap Q}\left|f_{n, 2}\right| d \mu=\frac{1}{\mu(Q)} \sum_{j \in J} \int_{Q_{j}}\left|f_{n, 2}\right| d \mu .
$$

Thus,

$$
\begin{aligned}
\frac{1}{\mu(Q)} \int_{Q}\left|f_{n, 2}(y)\right| d \mu(y) & =\frac{1}{\mu(Q)} \sum_{j \in J} \int_{Q_{j}}\left|f_{n}(y)\right| d \mu(y) \\
& =\frac{1}{\mu(Q)} \sum_{j \in J} \int_{Q_{j}}\left[\frac{1}{\mu\left(Q_{j}\right)} \int_{Q_{j}}\left|f_{n}(y)\right| d \mu(y)\right] d \mu(z) \\
& =\frac{1}{\mu(Q)} \sum_{j \in J} \int_{Q_{j}}\left|\tilde{f}_{n}(z)\right| d \mu(z)
\end{aligned}
$$




$$
\leq C \frac{1}{\mu(Q)} \int_{Q}\left|\tilde{f}_{n}(y)\right| d \mu(y) \leq C M_{\mathcal{D}} \tilde{f}_{n}(x),
$$

which proves (4.2).

Now, we write

$$
w\left(\left\{x \in X: I_{2}(x)>\lambda / 2\right\}\right) \leq w(\Omega)+w\left(\left\{x \in X \backslash \Omega: I_{2}(x)>\lambda / 2\right\}\right)=I+I I .
$$

To estimate $I$ we start estimating $w\left(Q_{j}\right)$. Let us first assume that $p>1$. From Hölder's inequality and since $w \in A_{p}^{\mathcal{D}}$ we have that

$$
\begin{aligned}
\lambda & <\frac{1}{\mu\left(Q_{j}\right)} \int_{Q_{j}}\|\mathbf{f}(x)\|_{\ell^{r}} d \mu(x) \\
& \leq \frac{1}{\mu\left(Q_{j}\right)}\left(\int_{Q_{j}}\|\mathbf{f}(x)\|_{\ell^{r}}^{p} w(x) d \mu(x)\right)^{1 / p}\left(\int_{Q_{j}} w(x)^{-p^{\prime} / p} d \mu(x)\right)^{1 / p^{\prime}} \\
& \leq C\left(\int_{Q_{j}}\|\mathbf{f}(x)\|_{\ell^{r}}^{p} w(x) d \mu(x)\right)^{1 / p}\left(\int_{Q_{j}} w(x) d \mu(x)\right)^{-1 / p} .
\end{aligned}
$$

Then

$$
w\left(Q_{j}\right) \leq C \frac{1}{\lambda^{p}} \int_{Q_{j}}\|\mathbf{f}(x)\|_{\ell^{r}}^{p} w(x) d \mu(x) .
$$

In the case $p=1$ using the $A_{1}^{\mathcal{D}}$ condition we get that

$$
w\left(Q_{j}\right)<\frac{1}{\lambda} \int_{Q_{j}}\|\mathbf{f}(x)\|_{\ell^{r}} \frac{w\left(Q_{j}\right)}{\mu\left(Q_{j}\right)} d \mu(x) \leq \frac{C}{\lambda} \int_{Q_{j}}\|\mathbf{f}(x)\|_{\ell^{r}} w(x) d \mu(x) .
$$

Therefore

$$
I=\sum_{j \in I} w\left(Q_{j}\right) \leq \sum_{j \in I} \frac{C}{\lambda^{p}} \int_{Q_{j}}\|\mathbf{f}(x)\|_{\ell^{r}}^{p} w(x) d \mu(x) \leq \frac{C}{\lambda^{p}} \int_{X}\|\mathbf{f}(x)\|_{\ell^{r}}^{p} w(x) d \mu(x) .
$$

The inequality $(a)$ for $p \leq r$ will be a consequence of the inequality $I I \leq c w(\Omega)$. In the sequel we shall write $\tilde{\mathbf{f}}$ for denote the sequence $\tilde{\mathbf{f}}=\left(\tilde{f}_{n}: n \in \mathbb{N}\right)$. Notice that from Theorem $4.2(f)$ we have

$$
\begin{aligned}
I I & \leq w\left(\left\{x \in X:\left\|M_{\mathcal{D}} \tilde{\mathbf{f}}(x)\right\|_{\ell^{r}}>\lambda / 2 C\right\}\right) . \\
& \leq \frac{2 C}{\lambda^{r}} \int_{X}\left\|M_{\mathcal{D}} \tilde{\mathbf{f}}(x)\right\|_{\ell^{r}}^{r} w(x) d \mu(x) . \\
& \leq \frac{C}{\lambda^{r}} \int_{X}\|\tilde{\mathbf{f}}(x)\|_{\ell^{r}}^{r} w(x) d \mu(x) \\
& =\frac{C}{\lambda^{r}} \int_{\Omega}\|\tilde{\mathbf{f}}(x)\|_{\ell^{r}}^{r} w(x) d \mu(x) .
\end{aligned}
$$

If we denote with $\tilde{Q}_{j}$ the first-ancestor of the cube $Q_{j} \in \mathcal{F}$ then, from Minkowski's inequality, for each $x \in \Omega$ we obtain that

$$
\begin{aligned}
\|\tilde{\mathbf{f}}(x)\|_{\ell^{r}} & =\left(\sum_{n \in \mathbb{N}}\left[\frac{1}{\mu\left(Q_{j}\right)} \int_{Q_{j}}\left|f_{n}(y)\right| d \mu(y)\right]^{r}\right)^{1 / r} \\
& \leq \frac{1}{\mu\left(Q_{j}\right)} \int_{Q_{j}}\|\mathbf{f}(y)\|_{\ell^{r}} d \mu(y)
\end{aligned}
$$




$$
\leq \frac{C}{\mu\left(\tilde{Q}_{j}\right)} \int_{\tilde{Q}_{j}}\|\mathbf{f}(y)\|_{\ell^{r}} d \mu(y) \leq C \lambda .
$$

Hence

$$
I I \leq \frac{C}{\lambda^{r}} \int_{\Omega} \lambda^{r} w(x) d \mu(x)=C w(\Omega),
$$

as desired.

Once some weak type inequalities are established, the arguments for the remaining inequalities no longer depend on the geometric nature of the domain of the function spaces. Hence the classical techniques ([5]) applies mutatis mutandi. We sketch them only for the sake of completeness.

Let us start proving $(b)$ for $1<p<r$. If $w \in A_{p}^{\mathcal{D}}$ then, from Theorem $4.2(c)$ and $(e)$, there exist $p_{1}$ and $p_{2}$ with $1 \leq p_{1}<p<p_{2}<r$ such that $w \in A_{p_{1}}^{\mathcal{D}}$ and $w \in A_{p_{2}}^{\mathcal{D}}$. Therefore $(a)$ holds for $p_{1}$ and $p_{2}$. Then from Lemma 4.4 we obtain $(b)$ for every $p$ such that $p_{1}<p<p_{2}$, and therefore $(b)$ holds for $1<p<r$.

The proof of $(b)$ in the case $p>r$ is obtained from a duality argument if $r$ is small enough and by Lemma 4.5 otherwise. First notice that since $w \in A_{p}^{\mathcal{D}}$ with $p>r$, by Theorem $4.2(e), w \in A_{\frac{p}{r_{0}}}^{\mathcal{D}}$ for some $1<r_{0}<p$. If $r_{0}<r<p$, since $(b)$ holds for $r=p$, we only have to obtain (b) for $r_{0}$ and apply Lemma 4.5. So that we only need to prove $(b)$ for $1<r \leq r_{0}$.

Since $w \in A_{\frac{p}{r_{0}}}^{\mathcal{D}}$, from Theorem $4.2(c), w \in A_{q}^{\mathcal{D}}$ for every $q \geq \frac{p}{r_{0}}$ and from Theorem $4.2(d)$ and $(a)$, we obtain that $w^{1-q^{\prime}} \in A_{q^{\prime}}^{\mathcal{D}}$ and $w(x)>0$ almost everywhere. Let $\varphi \geq 0$ be such that $\varphi \in L_{w}^{q^{\prime}}(X, \mu)$ with $\|\varphi\|_{q^{\prime}, w} \leq 1$. From the dyadic weighted inequality for $M_{\mathcal{D}}$ we obtain

$$
\int_{X}\left|M_{\mathcal{D}}(\varphi w)\right|^{q^{\prime}} w(x)^{1-q^{\prime}} d \mu(x) \leq C \int_{X}|\varphi w|^{q^{\prime}} w(x)^{1-q^{\prime}} d \mu(x) \leq C .
$$

Now, from Proposition 4.6 and Hölder's inequality with $q$ and $q^{\prime}$

$$
\begin{aligned}
\int_{X}\left\|M_{\mathcal{D}} \mathbf{f}(x)\right\|_{\ell^{r}}^{r} \varphi(x) w(x) d \mu(x) & \leq C \int_{X}\|\mathbf{f}(x)\|_{\ell^{r}}^{r} M_{\mathcal{D}}(\varphi w)(x) d \mu(x) \\
& \leq C D\left(\int_{X}\|\mathbf{f}(x)\|_{\ell^{r}}^{r q} w(x) d \mu(x)\right)^{1 / q},
\end{aligned}
$$

with

$$
\begin{aligned}
D & =\left(\int_{X}\left(\frac{M_{\mathcal{D}}(\varphi w)(x)}{w(x)}\right)^{q^{\prime}} w(x) d \mu(x)\right)^{1 / q^{\prime}} \\
& =\left(\int_{X}\left(M_{\mathcal{D}}(\varphi w)(x)\right)^{q^{\prime}} w(x)^{1-q^{\prime}} d \mu(x)\right)^{1 / q^{\prime}} \leq C,
\end{aligned}
$$

for every $q \geq \frac{p}{r_{0}}$ and every $\varphi \geq 0$ such that $\|\varphi\|_{q^{\prime}, w} \leq 1$. Then, taking supremum over such $\varphi$ we obtain

$$
\left(\int_{X}\left\|M_{\mathcal{D}} \mathbf{f}(x)\right\|_{\ell^{r}}^{r q} w(x) d \mu(x)\right)^{1 / q} \leq C\left(\int_{X}\|\mathbf{f}(x)\|_{\ell^{r}}^{r q} w(x) d \mu(x)\right)^{1 / q},
$$

for every $q \geq \frac{p}{r_{0}}$. On the other hand since $1<r \leq r_{0}$, then the above inequality holds with $q=p / r \geq p / r_{0}$, which is the desired inequality in $(b)$. 


\section{REFERENCES}

[1] H. Aimar, Construction of Haar type bases on quasi-metric spaces with finite Assouad dimension, Anal. Acad. Nac. Cs. Ex., F. y Nat., Buenos Aires 54 (2004).

[2] H. Aimar, A. Bernardis and B. Iaffei, Comparison of Hardy-Littlewood and dyadic maximal functions on spaces of homogeneous type,J. Math. Anal. Appl., 312 (2005) 105-120.

[3] H. Aimar, A. Bernardis and B. Iaffei, Multiresolution approximation and unconditional bases on weighted Lebesgue spaces on spaces of homogeneous type, J. Approx. Theory, 148 (2007) 12-34.

[4] H. Aimar, A. Bernardis and L. Nowak Equivalence of Haar bases associated to different dyadic systems, In Press in Journal of Geometric Analysis.

[5] K. Andersen and R. John, Weighted inequalities for vector-valued maximal functions and singular integrals, Studia Math. T. LXIX, (1980), 19-31.

[6] A. Benedek, A. P. Calderón and R. Panzone, Convolution operators on Banach space valued functions, Proc. Nat. Acad. Sc. U.S.A. 48 (1962), 356-365.

[7] A. Benedek and R. Panzone, The spaces $L^{p}$ withmixed norm, Duke Math. J. 28 (1961), 301-324.

[8] A. P. Calderón and A. Zygmund, A note on the interpolation of sublinear operations, Amer. J. Math. 78 (1956), 282-288.

[9] M. Christ, A T(b) theorem with remarks on analytic capacity and the Cauchy integral, Colloq. Math. 60/61 (2) (1990), 601-628.

[10] C. Fefferman and E. M. Stein, Some maximal inequalities. Amer. J. Math. 93 (1971), $107-115$.

[11] I. Genebashvili, A. Gogatishvili, V. Kokilashvili and M. Krbec, Weight theory for integral transforms on space of homogeneous type, Addison Wesley Longman Limited. 1998.

[12] S. Konyagin and V. Temlyakov, A remark on greedy approximation in Banach spaces., East Journal on Approx., 5(3)(1999), 365-379.

[13] R. Macias and C. Segovia, Lipschitz functions on spaces of homogeneous type. Adv. in Math. 33 (1979), 271-309.

[14] K. Parthasarathy, Introduction to probability and measure, Springer-Verlag, New York 1978.

[15] R. Toledano, Desigualdades de Harnack elíptica y parabólica, un enfoque abstracto, Tesis doctoral. FIQ-UNL. 1999.

[16] S. Treil and A. Volberg, Wavelets and the angle between past and future, J. Funct. Anal. 143 (1997), 269-308.

[17] I. Verbitsky, Imbedding and multiplier theorems for discrete Littlewood-Paley spaces. Pacific J. Math. 176 (1996), 529-556.

[18] R. Young, An introduction to nonharmonic Fourier series, Academic Press. 1980.

Departamento de Matemática (FIQ-UNL); IMAL-COniCET, Santa Fe, Argentina

E-mail address: haimar@santafe-conicet.gov.ar

Departamento de Matemática (FIQ-UNL); IMAL-COniCET, Santa Fe, Argentina

E-mail address: bernard@santafe-conicet.gov.ar

Departamento de Matemtica (FaEA-UnComa), Neuquen; Imal-Conicet, Santa Fe, Argentina

E-mail address: luisenlitoral@yahoo.com.ar 